\title{
Central Nervous System
}

National Cancer Institute

\section{Source}

National Cancer Institute. Central Nervous System. NCI Thesaurus. Code C12438.

The part of the nervous system that consists of the brain, spinal cord, and meninges. 